\title{
Semiconductor and Metal Nanoparticle Formation on Polymer Spheres Coated with Weak Polyelectrolyte Multilayers
}

\author{
Peter Schuetz ${ }^{1}$ and Frank Caruso ${ }^{*}$ \\ ${ }^{1}$ Max Planck Institute of Colloids and Interfaces, D-14424 Potsdam, Germany, and \\ ${ }^{2}$ Centre for Nanoscience and Nanotechnology, Department of Chemical and Biomolecular \\ Engineering, The University of Melbourne, Victoria 3010, Australia
}

Supporting Information

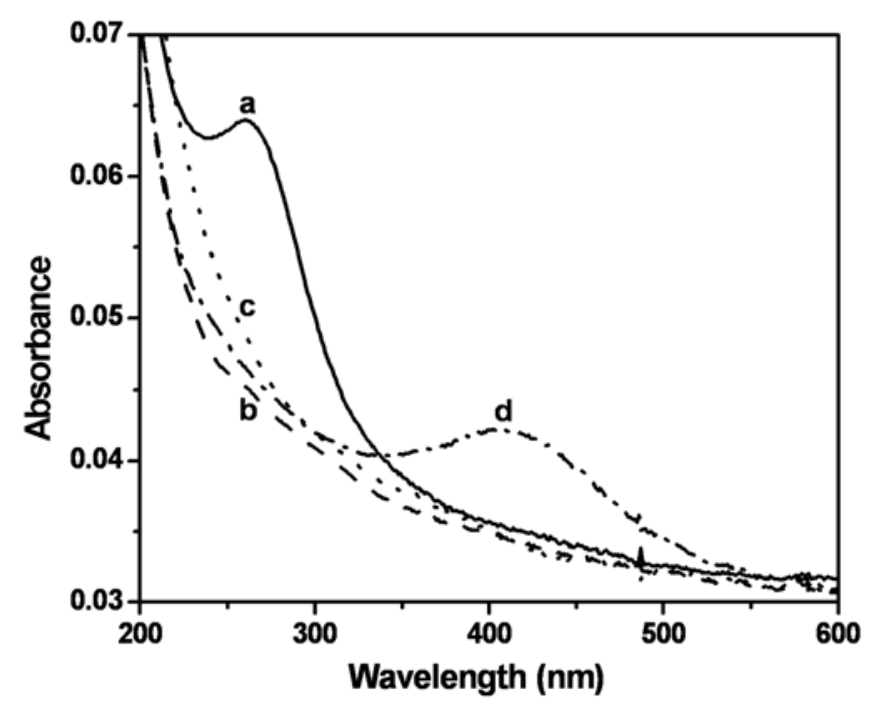

Figure S1. UV-vis-spectra for the formation of Ag NPs in PAH/PAA multilayers deposited onto quartz substrates. The final film structure is PEI/(PAH/PAA) $4 / \mathrm{PAH} / \mathrm{PSS} / \mathrm{PDDA} / \mathrm{PSS}$. Spectra for films with complexed copper ions (1) are shown, as well as after copper release (2), adsorption of silver ions (3), and reduction with $\mathrm{NaBH}_{4}$ (4). The Ag NPs were not stable under normal conditions and the plasmon peak vanished after 3 to $4 \mathrm{~h}$. 


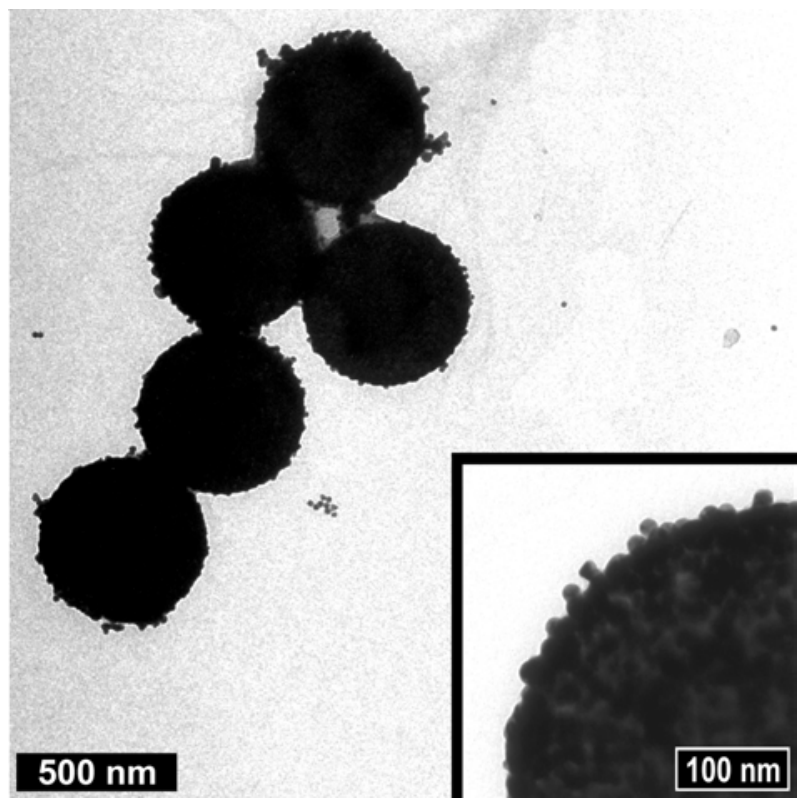

Figure S2. TEM images of electroless plated, gold NP-seeded coatings on $496 \mathrm{~nm}$ diameter PS spheres. In the first step, gold NPs were formed in situ on the spheres by loading the PAH/PAA multilayers with $\mathrm{AuCl}_{4}{ }^{-}$and reacting it with $\mathrm{NaBH}_{4}$. (The film structure is (PAH/PAA) $)_{4} / \mathrm{PAH} / \mathrm{PSS} / \mathrm{PDDA} / \mathrm{PSS}$.) A gold plating solution was then added to the dispersion of gold NP-seeded particles to increase the gold content in the coating. (See text for details.) 